\title{
Lifetime risks, loss of life expectancy, and health care expenditures for 19 types of cancer in Taiwan
}

This article was published in the following Dove Press journal:

Clinical Epidemiology

\section{Tzu-Yi Wu' \\ Chia-Hua Chung ${ }^{2}$ \\ Chia-Ni Lin ${ }^{3}$ \\ Jing-Shiang Hwang ${ }^{2}$ \\ Jung-Der Wang $g^{3,4}$}

'Institute of Economics, Academia Sinica, Taipei, Taiwan; ${ }^{2}$ Institute of Statistical Science, Academia Sinica, Taipei, Taiwan; ${ }^{3}$ Department of Public Health, College of Medicine, National Cheng Kung University, Tainan, Taiwan ${ }^{4}$ Department of Occupational and Environmental Medicine, National Cheng Kung University Hospital, College of Medicine, National Cheng Kung University, Tainan, Taiwan

\section{Video abstract}

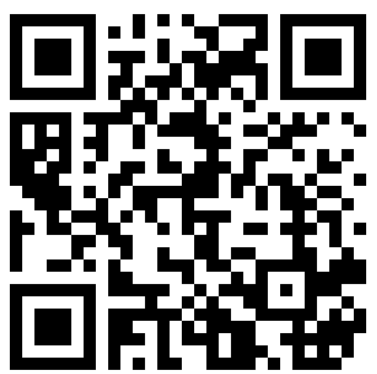

Point your SmartPhone at the code above. If you have a QR code reader the video abstract will appear. Or use: http://youtu.be/sWAG0]x7Pq4

Correspondence: Jung-Der Wang Department of Public Health, College of Medicine, National Cheng Kung University, No. I, University Road, East District, Tainan 70I, Taiwan

Tel +886 62353535 ext 5600

Fax +88662359033

Email jdwang121@gmail.com
Background: The mortality rates for different cancers are no longer an efficient tool for making national policy. The purpose of this study were to quantify the lifetime risks, life expectancies (LEs) after diagnosis, expected years of life lost (EYLL), and lifetime health care expenditures for 19 major cancers in Taiwan.

Methods: A total of 831,314 patients with 19 pathologically proven cancers were abstracted from the Taiwan Cancer Registry from 1998 to 2012. They were linked to the National Mortality Registry (1998-2014) and National Health Insurance reimbursement database (1998-2013) for survival and health care costs. We estimated the cumulative incidence rate for ages 0-79 years and the lifetime survival function for patients with different cancer sites. The EYLL was calculated by subtracting the LE of each cancer cohort from that of the age- and sex-matched referents simulated from national life tables. The estimated lifetime cost was calculated by adding up the product of survival probability and mean cost at the corresponding duration-to-date after adjustment for the inflation to the year of 2013.

Results: There were 5 cancers with a lifetime risk exceeding 4\%: colorectal, liver, lung, and prostate in males, and breast and colorectal in females. Cancers with EYLL of $>10$ years were: esophageal, intrahepatic bile ducts, liver, pancreas, oral, nasopharyngeal, leukemia, lung, and gallbladder, extrahepatic bile ducts and biliary tract in males, and intrahepatic bile ducts, pancreas, nasopharyngeal, lung, esophageal, leukemia, liver, gallbladder, extrahepatic bile ducts and biliary tract, ovary, and stomach in females. Cancers with lifetime health care expenditures exceeding US\$50,000 to the National Health Insurance were as follows: leukemia, kidney, testis, renal pelvis and ureter in males, and renal pelvis and ureter, leukemia, breast, urinary bladder, kidney, ovary, and nasopharyngeal in females. All these impacts should be considered in health policy decisions.

Conclusion: The impacts of cancer in Taiwan are very large. Future studies must consider both quality of life and the entire impact from societal perspectives.

Keywords: health impacts, incidence rate, expected years of life lost, cancer prevention

\section{Introduction}

Cancer is one of the leading causes of death in the world ${ }^{1}$ although the momentum of this disease has slowed down slightly in some developed countries due to cancer prevention strategies and action programs, early diagnosis, and treatment. ${ }^{2}$ In many developing countries, however, the trends of a growing cancer burden continue and are projected to increase in the coming decades due to population growth and aging. ${ }^{3}$ In the USA, it is estimated that there were $15,500,000$ cancer survivors in 2016, a number, which is expected to increase to over $20,000,000$ by $2026 .{ }^{4}$ In Taiwan, there were 431,401 cancer survivors who were registered as having a catastrophic illness 
and required health care in 2017 , which is $>1.5$ times the number in 2003. 5,6 The societal costs of cancer, including at least losses of life-years, functional disability, and health care expenditures, deserve quantification for a national cancer control plan. ${ }^{7}$

The current trend of quantification for the burden of cancer usually focuses on estimation of disability-adjusted life years, which is composed of years of life lost (YLL) and the years lost due to disability. ${ }^{8}$ The related studies have been quite useful to obtain a quick, ballpark view of the global burden, especially when cancer registries in many countries are still at their initial or developing stages. In countries where big data regarding long-term follow-up are accessible, direct estimation of the difference in life expectancy (LE) between patients with a specific disease or cancer and those of age- and sex-matched referents simulated from national life tables, or, expected years of life lost are feasible. ${ }^{9,10}$ Moreover, inter-linkages of these data files can provide opportunities for estimation of lifetime health care expenditures and the demand for long-term care. ${ }^{11-13}$ The development of these novel methods and big data linkages would make possible a more comprehensive estimation of the societal impact of a specific illness, ${ }^{7}$ including cancer.

In this study, we aim to inter-link 4 population-based data files of the Taiwan Cancer Registry, the Taiwan National Vital Statistics, the National Health Insurance (NHI), and the Taiwan Mortality Registry, to quantify lifetime risk (or, cumulative incidence rate from ages $\left.0-79\left[\mathrm{CIR}_{0-79}\right]\right)$, LE after diagnosis, expected years of life lost (EYLL), and lifetime health care expenditures reimbursed by NHI for 19 major cancers followed for 17 years. As a corollary product, we also estimate the cost-per-life-year for cancer care, a generalized cost-effectiveness assessment. The analysis would be useful for resource allocation of cancer control for health policy makers and/or to help in making a direct comparison between the prevention and treatment of different cancers.

\section{Methods}

The study was approved by the Institutional Review Board of National Cheng Kung University Hospital (IRB number: A-ER-103-203) before commencement.

\section{Study population and datasets}

A total of 831,314 patients with a pathologically validated cancer diagnosis when aged between 0 and 79 years old were abstracted and interlinked from 4 datasets, as summarized in Figure 1: the Taiwan Cancer Registry for 1998-2012, the Taiwan National Vital Statistics for 1998-2012, the reimbursement database of the NHI system (1998-2013), and the Taiwan Mortality Registry (1998-2014). These datasets contain data on the patients' demographics, date of diagnosis, cancer site, histology, national life tables, survival status, and health care expenditure covered by the NHI. Because cancer is a catastrophic illness, the Taiwan NHI covers all costs of outpatient clinic visits and hospitalization, including laboratory and imaging studies and prescription medicines. The patients' personal information was protected by encrypting their identification numbers. The 19 major cancers included liver (ICD9-CM code: 155), lung (ICD-9-CM code: 162), colorectal (ICD-9-CM code: 153-154, except for 153.5, 154.2-154.3, 154.8), oral (ICD-9-CM code: 140-141, 143-145), leukemia (ICD-O-3 morphology code: 9590-9729, 9731-9734, 9800-9805, 9820-9837, 9840-9930, 9940, 9950-9964, 9975, except for 9832-9834, 9870, 9931), stomach (ICD-9-CM code: 151.0-151.9), nasopharynx (ICD-9-CM code: 147.0-147.9), prostate (ICD-9-CM code: 185), urinary bladder (ICD-9-CM code: 188.0-188.9), kidney (ICD-9-CM code: 189.0), renal pelvis and ureter (ICD-9-CM code: 189.1-189.2), testis (ICD-9-CM code: 186.0-186.9), breast (ICD-9-CM code: 174.0-174.9), ovary (ICD-9-CM code: 183.0-183.9), cervix (ICD-9-CM code: 180.0-180.9), esophagus (ICD-9-CM code: 150.0-150.9), intrahepatic bile ducts (ICD-9-CM code: 155.1), gallbladder, extrahepatic bile ducts and biliary tract (ICD-9-CM code: 156.0-156.1, 156.9), and pancreas (ICD9-CM code: 157.0-157.2, 157.8). The patients with cancer of the same organ system were grouped as a dynamic cancer cohort, indicating that they were diagnosed and recruited into the cohort at different calendar years during the period from 1998 to 2012, and we collected a total of 19 cancer cohorts.

\section{Estimation of lifetime risk by CIR}

With the age- and sex-specific number of new cases from the Taiwan Cancer Registry for every 3 consecutive calendar years as the numerators, and the corresponding population at risk from vital statistics as the denominators, we calculated the age- and sex-specific incidence rates (IR) and CIR for the 19 cancers. The CIR was calculated from ages 0 to $79\left(\mathrm{CIR}_{0-79}\right)$ to estimate the lifetime risk of a specific cancer. The $\mathrm{CIR}_{0-79}$ was calculated from 1998 to 2012 to determine the trends in changes of lifetime risks. The formula is as follows: ${ }^{14}$

$\underset{70-79}{\mathrm{CIR}_{0-79}}=1-\exp \left(-\sum_{\mathrm{i}}\left(\operatorname{IR}_{\mathrm{i}}\right)\left(\Delta \mathrm{t}_{\mathrm{i}}\right)\right)$, where $i=0-9,10-19, \ldots$,

where $I R_{i}$ is incidence rate for the $i$-th age group and $\Delta t_{i}=10$ years of age range. 


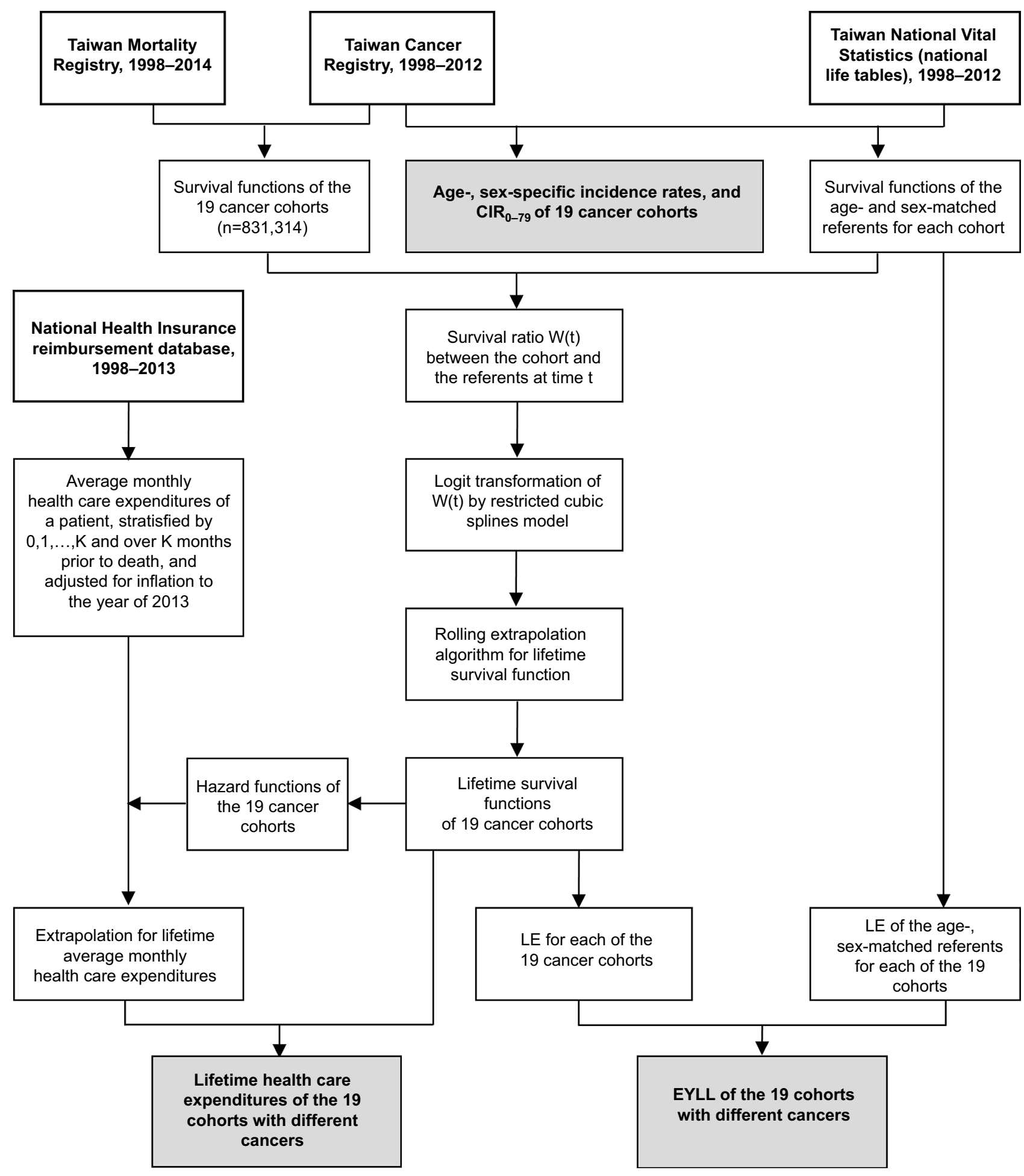

Figure I Flow diagram of the inclusion of subjects and their relevant information for estimations.

Notes: Bold type indicates original datasets, while with shading indicates results.

Abbreviations: $\mathrm{CIR}_{0-79}$, cumulative incidence rate, aged 0-79 years; EYLL, expected years of life lost; LE, life expectancy.

\section{Estimation of lifetime survival function, LE after diagnosis, and EYLL}

Each cancer patient's survival state was ascertained by linkage to the National Mortality Registry during 1998-2014.
The survival functions were produced via the Kaplan-Meier estimation method until the end of the follow-up. Further extrapolation of survival function was estimated using a rolling extrapolation algorithm, aided by the age- and 
sex-matched referents simulated from the National Vital Statistics life tables. ${ }^{11}$ The EYLL was the difference between LEs of the cancer cohort and those of the age- and sex-matched referents (Supplementary materials). The iSQoL 2 software was used to compute these estimations. ${ }^{15}$

\section{Estimation of average monthly cost and its extrapolation to lifetime}

Taiwan's NHI categorizes every cancer patient with pathological proof as a case of catastrophic illness. The applications must be validated by 2 physicians and can then be waived from all co-payments related to the specific cancer or illness. Therefore, our reimbursement data for cancer are very comprehensive and cover all health care expenditures related to the registered illness. We added all the reimbursement costs for every case and calculated the monthly average after diagnosis. The estimated lifetime cost was calculated by adding the product of monthly survival rates and monthly mean costs. ${ }^{11}$ The monthly mean costs beyond the maximum follow-up were estimated using the mean expenditures of the patients in a specific number of months prior to their death with a weighted average. The number could be decided according to the observed costs in the patients' last months, where the weights were dependent on the extrapolated hazard of death. The estimates of lifetime costs were also calculated using the iSQoL2 package.

\section{Results}

The demographic data and burdens to health $\left(\mathrm{CIR}_{0-79}, \mathrm{LE}\right.$ after diagnosis, and EYLL) and lifetime costs of the 19 cancers are summarized in Table 1. In males, the number of patients with liver cancer was the highest. Patients with prostate cancer showed the highest mean age at diagnosis (73.4 \pm 8.5 years). In females, the cohort of patients with breast cancer was the largest. Patients with cancers of the gallbladder, extrahepatic bile ducts, and biliary tract showed the highest mean age at diagnosis $(68.9 \pm 12.5$ years $)$.

The lifetime risks of colorectal cancer and breast cancer were the highest, with $\mathrm{CIR}_{0-79}$ of $6.79 \%$ and $6.77 \%$, among males and females, respectively, from 2010 to 2012 . The highest EYLL in males and females were esophageal cancer and cancer of intrahepatic bile ducts, with estimates of 19.1 and 17.1 years, respectively. Leukemia and cancer of renal pelvis and ureter represented the highest lifetime costs in males and females, with estimates of US\$67,388 and US\$78,586, respectively.

Figure 2 summarizes the dynamic changes in $\mathrm{CIR}_{0-79}$ for different cancers in both genders in the period from
1998 to 2012. In males, colorectal cancer surpassed cancers of the liver and lung to become the highest $\mathrm{CIR}_{0-79}$ after 2010. While prostate cancer, oral cancer, esophageal cancer, and leukemia show an increasing trend, those of stomach and urinary bladder seem to have decreased over the last one and half decades. In addition, the trends of the other cancers appear flattened. In females, cancers of breast, colorectum, and lung show the highest $\mathrm{CIR}_{0-79}$ and a generally increasing trend. Among these, the lifetime risk of breast cancer rose very fast, from $3.80 \%$ to $6.77 \%$, during the observation period. In contrast, cervical cancer dramatically decreased, from a $\mathrm{CIR}_{0-79}$ of $3.04 \%$ down to $1.17 \%$. The trends of changes in $\mathrm{CIR}_{0-79}$ among other cancers in females were similar to those seen in males, except for lung cancer, which still exhibited a rising trend.

Figure 3 shows the EYLL and lifetime costs of the 19 cancers under consideration in Taiwan, stratified by gender. Leukemia seems to have the highest health burden and health care expenditures in both males and females, with an EYLL of $>10$ years and a lifetime cost of $>$ US $\$ 50,000$.

Figure 4 shows the cost-effectiveness of health care services for cancer. By dividing the lifetime cost by the LE of the cancer of a specific organ system, we were able to estimate the cost-per-life year survival. Testicular and cervical cancers had the least steep slopes in males and females, respectively. In contrast, pancreas cancer had the steepest slopes in both males and females.

\section{Discussion}

This study links 4 national datasets to quantify the likelihood and major consequences of common cancers with a lifetime horizon. Namely, we estimated the lifetime risk, EYLL, and health care expenditures for 19 major cancers in Taiwan. The following arguments corroborate the accuracy of these estimates: first, the 4 datasets are well validated. Take the National Cancer Registry as an example. Taiwan established this system in 1979 and required all hospitals to report. ${ }^{16,17}$ All diagnoses require pathological evidence, with the exception of liver cancer, and all cancers had to be validated by at least 2 qualified experts after implementation by the NHI in 1995 (Supplementary materials). ${ }^{17,18}$ Second, we followed 19 groups of patients with different cancer sites from 1998 to 2014 , or, a total of 17 years. Therefore, we covered the survival function and reimbursement data up to the LE of most of such cancers. A long-term follow-up would be helpful to decrease the effects of individual fluctuations. Third, the rolling extrapolation method extended the survival month by month until the survival rate was $<0.1 \%$, which usually leads 
Table I Comparison of frequency distributions and expected lifetime impacts for 19 cancers in Taiwan stratified by gender: LE after diagnosis, EYLL, lifetime cost, $\mathrm{CIR}_{0-79}$, and $95 \% \mathrm{Cl}$

\begin{tabular}{|c|c|c|c|c|c|c|}
\hline Cancer & $\mathbf{N}$ & $\begin{array}{l}\text { Age (years) } \\
(\text { mean } \pm \text { SD) }\end{array}$ & $\begin{array}{l}\mathrm{CIR}_{0-79}{ }^{\mathrm{a}} \\
(\%)\end{array}$ & $\begin{array}{l}\text { LE after diagnosis } \\
\text { ( } 95 \% \mathrm{Cl} \text {, years) }\end{array}$ & $\begin{array}{l}\text { EYLL }(95 \% \mathrm{Cl}) \\
\text { (years) }\end{array}$ & $\begin{array}{l}\text { Lifetime cost } \\
\left(95 \% \mathrm{Cl}, \times 10^{3} \text { USD) }\right.\end{array}$ \\
\hline \multicolumn{7}{|l|}{ Male } \\
\hline Liver & 99,659 & $60.9 \pm 13.4$ & 6.66 & $4.7(4.6-4.8)$ & $16.0(15.8-16.1)$ & $23.0(22.4-23.6)$ \\
\hline Lung & 82,820 & $68.9 \pm 12.0$ & 6.31 & $2.6(2.5-2.7)$ & $12.6(12.5-12.7)$ & $2 \mid .4(2||-2 \mid .8)$. \\
\hline Colorectum & 80,425 & $65.7 \pm 13.3$ & 6.79 & $10.9(10.7-11.1)$ & $6.5(6.3-6.7)$ & $40.4(39.7-4 \mid .2)$ \\
\hline Oral & 44,089 & $52.4 \pm 11.8$ & 2.85 & $12.2(11.7-12.6)$ & $15.0(14.5-15.4)$ & $48.7(47.3-50.1)$ \\
\hline Esophagus & 22,504 & $59.1 \pm 12.4$ & 1.64 & $3.1(2.9-3.3)$ & $19.1(18.8-19.3)$ & $25.9(25.3-26.6)$ \\
\hline Leukemia & 30,823 & $56.7 \pm 21.8$ & 2.05 & $12.0(11.3-12.6)$ & $13.0(\mid 2.4-13.6)$ & $67.4(64.5-70.2)$ \\
\hline Stomach & 34,726 & $68.2 \pm 13.3$ & 2.07 & $6.2(6-6.4)$ & $9.5(9.3-9.7)$ & $25.6(24.9-26.3)$ \\
\hline Nasopharynx & 16,758 & $50.3 \pm 13.1$ & 0.85 & $14.3(13.5-15.2)$ & $14.4(\mid 3.6-15.3)$ & $48.9(45.8-52.0)$ \\
\hline Pancreas & 9,057 & $68.9 \pm 12.5$ & 0.78 & $1.6(I-2.1)$ & $15.2(15-15.4)$ & $20.3(10.6-30.1)$ \\
\hline Prostate & 46,107 & $73.4 \pm 8.5$ & 4.83 & $9.7(9.5-9.8)$ & $2.4(2.3-2.6)$ & $40.6(39.6-41.5)$ \\
\hline Intrahepatic bile ducts & 5,994 & $68.4 \pm 12.3$ & 0.53 & $1.9(1.5-2.3)$ & $16.0(15.8-16.3)$ & $15.4(14.5-16.3)$ \\
\hline Bladder & 20,360 & $68.5 \pm 12.9$ & 1.29 & $11.0(10.6-11.4)$ & $4.6(4.2-4.9)$ & $42.3(40.8-43.7)$ \\
\hline Kidney & 7,510 & $60.7 \pm 15.9$ & 0.62 & $13.5(\mid 2.5-14.5)$ & $7.7(6.7-8.7)$ & $59.9(54.5-65.2)$ \\
\hline Renal pelvis and ureter & 6,425 & $66.4 \pm 12.3$ & 0.55 & $9.1(8.6-9.7)$ & $7.7(7.2-8.3)$ & $54.0(50.4-57.6)$ \\
\hline Gallbladder and extrahepatic bile ducts & 3,096 & $67.3 \pm 13.9$ & 0.24 & $3.2(2.3-4)$ & $12.3(11.8-12.8)$ & $20.3(|9.2-2| .4)$ \\
\hline Testis & 2,089 & $29.8 \pm 14.8$ & 0.07 & $42.7(39.4-45.9)$ & $4.2(1.0-7.5)$ & $59.7(50.9-68.4)$ \\
\hline \multicolumn{7}{|l|}{ Female } \\
\hline Lung & 43,569 & $65.9 \pm 13.3$ & 3.47 & $4.9(4.6-5.2)$ & $15.1(14.8-15.4)$ & $30.9(29.6-32.3)$ \\
\hline Breast & 101,550 & $52.4 \pm 12.2$ & 6.77 & $23.7(23.2-24.1)$ & $7.5(7.1-8.0)$ & $69.9(67.3-72.4)$ \\
\hline Liver & 37,328 & $66.9 \pm 12.4$ & 2.80 & $4.9(4.8-5.1)$ & $14.0(13.8-14.1)$ & $23.3(22.5-24.0)$ \\
\hline Colorectum & 60,075 & $65.1 \pm 14.2$ & 4.47 & $13.1(12.9-13.3)$ & $7.6(7.4-7.9)$ & $42.3(4 I .4-43.3)$ \\
\hline Leukemia & 22,322 & $55.4 \pm 21.2$ & 1.88 & $15.2(14.4-16)$ & $14.1(13.3-14.9)$ & $72.2(68.4-76.0)$ \\
\hline Stomach & 18,752 & $65.3 \pm 15.3$ & 1.09 & $9.0(8.5-9.4)$ & $11.7(11.2-12.1)$ & $29.3(27.8-30.7)$ \\
\hline Ovary & 14,494 & $51.1 \pm 15.7$ & 0.84 & $19.9(19-20.7)$ & $12.7(11.8-13.6)$ & $53.9(50.7-57.0)$ \\
\hline Pancreas & 6,350 & $68.1 \pm 13.1$ & 0.56 & $1.8(1.5-2.1)$ & $16.5(16.1-16.9)$ & $14.6(13.9-15.3)$ \\
\hline Cervix & 31,613 & $56.2 \pm 14.6$ & 1.17 & $21.3(20.9-21.7)$ & $6.4(6.0-6.8)$ & $45.6(44.2-46.9)$ \\
\hline Intrahepatic bile ducts & 5,219 & $67.0 \pm 12.1$ & $0.4 I$ & $1.8(1.6-2.1)$ & $17.1(16.8-17.4)$ & $14.5(\mid 3.6-15.4)$ \\
\hline Renal pelvis and ureter & 7,547 & $68.7 \pm 11.2$ & 0.61 & $10.2(9.6-10.8)$ & $7.3(6.7-7.9)$ & $78.6(72.4-84.7)$ \\
\hline Nasopharynx & 5,591 & $49.7 \pm 13.7$ & 0.25 & I8.3 (I6.I-20.4) & $15.2(13.0-17.4)$ & $5 I . I(46 . I-56.0)$ \\
\hline Bladder & 8,034 & $68.4 \pm 12.7$ & 0.49 & $10.9(\mid 0.4-11.4)$ & $7.0(6.5-7.5)$ & $69.2(64.3-74.0)$ \\
\hline Oral & 4,692 & $60.0 \pm 15.3$ & 0.34 & $15.0(13.6-16.4)$ & $10.0(8.6-11.3)$ & $44.8(40.1-49.6)$ \\
\hline Gallbladder and extrahepatic bile ducts & 3,002 & $68.9 \pm 12.5$ & 0.22 & $3.8(3.2-4.3)$ & $13.8(\mid 3.1-14.4)$ & $19.9(|8.5-2| .2)$ \\
\hline Kidney & 3,956 & $60.1 \pm 16.9$ & 0.30 & $15.9(\mid 4.3-17.5)$ & $9.0(7.4-10.6)$ & $56(47.8-64.1)$ \\
\hline Esophagus & $\mathrm{I}, 755$ & $67.3 \pm 13.9$ & 0.12 & $4.3(3.5-5.2)$ & $14.7(13.7-15.7)$ & $23.6(21.2-26.1)$ \\
\hline
\end{tabular}

Notes: ${ }^{\mathrm{a}} \mathrm{CIR}_{0-79}$ in 2010-2012.

Abbreviations: $\mathrm{CIR}_{0-79}$, cumulative incidence rate, aged 0-79; EYLL, expected years of life lost; LE, life expectancy.

to a more accurate estimation of the lifetime survival function than that obtained using the usual parametric method. ${ }^{11,15}$ Although our method may be more accurate than other currently available parametric methods, the estimation must be conducted again when there is any new development in treatment technology.

$\mathrm{CIR}_{0-79}$ can be regarded as the lifetime risk of disease occurrence. ${ }^{19}$ We found that colorectal and breast cancers had the highest $\mathrm{CIR}_{0-79}$ in males and females $(6.79 \%$ and $6.77 \%$, respectively), and their trends over the last 15 years still appeared to be rising at the end of 2012. Moreover, the trends in the IR of breast cancer and cervical cancer appear to be consistent with those in the USA and Korea. ${ }^{20,21}$ Through cancer screening, patients can detect their cancers at earlier stages, even at a premalignant stage, which would likely save life-years and health care costs, as demonstrated for colorectal and cervical cancers. ${ }^{22-24}$ Thus, Taiwan has launched a national campaign for screening of 4 cancers since $2010 .{ }^{25}$ The cervical cancer screening rate has been consistently high (over 50\% from 2004 to 2013), which may have contributed to the lowering of the $\mathrm{CIR}_{0-79}$ values seen in Figure $2 .{ }^{26}$ However, the screening rates of breast and colorectal cancers began to rise after 2010 but were still below $40 \%$ before 2012 and appeared to have no direct linear correlation with their individual $\mathrm{CIR}_{0-79}$ values (Figures S1 and S2). ${ }^{27,28}$ More research is needed to explore the related risk factors and determine the optimal age of beginning such screenings for a more cost-effective national strategy. 

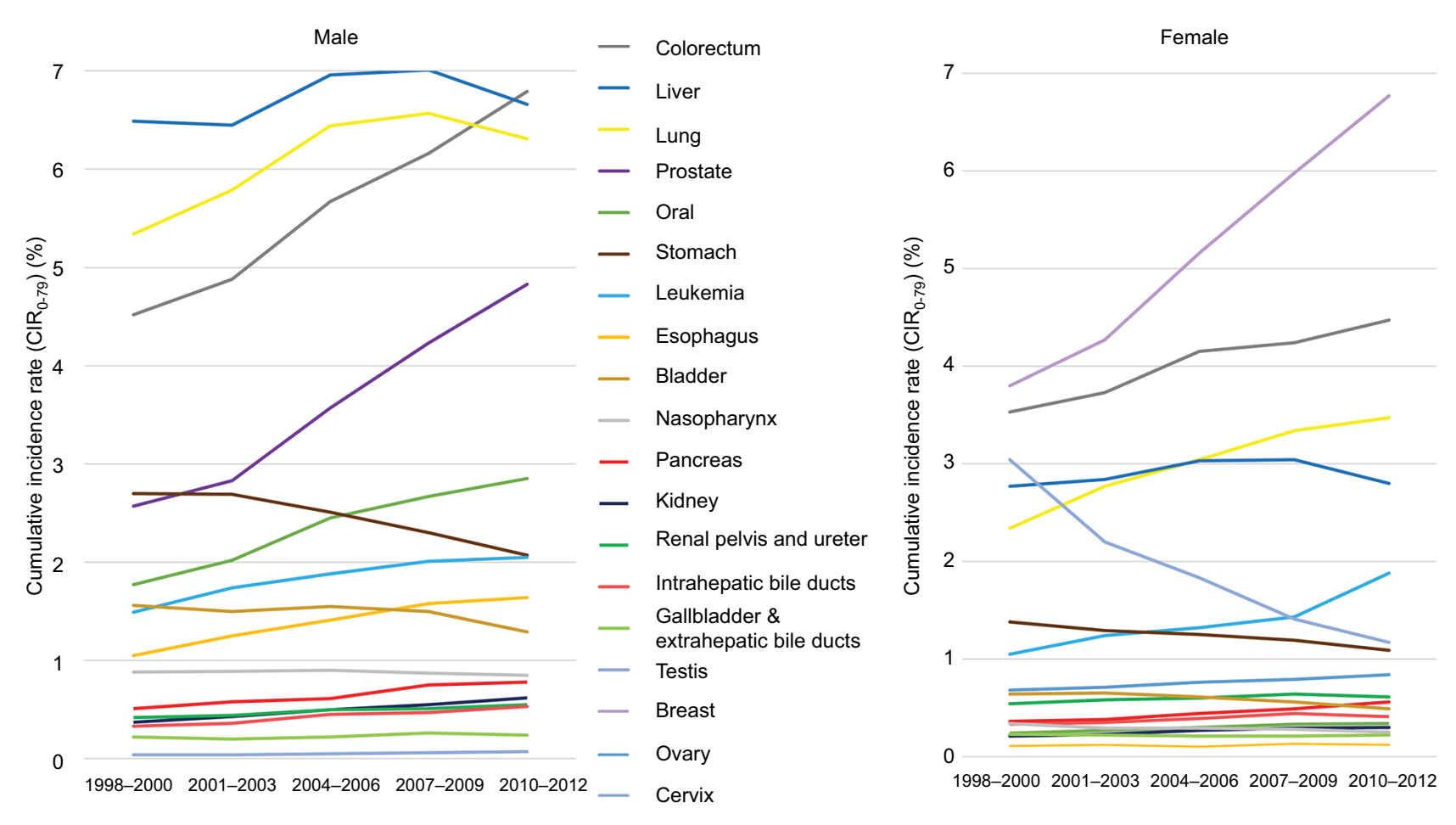

Figure $2 \mathrm{ClR}_{0-79}$ along a calendar period.

Abbreviations: $\mathrm{CIR}_{0-79}$, cumulative incidence rate, aged 0-79 years.
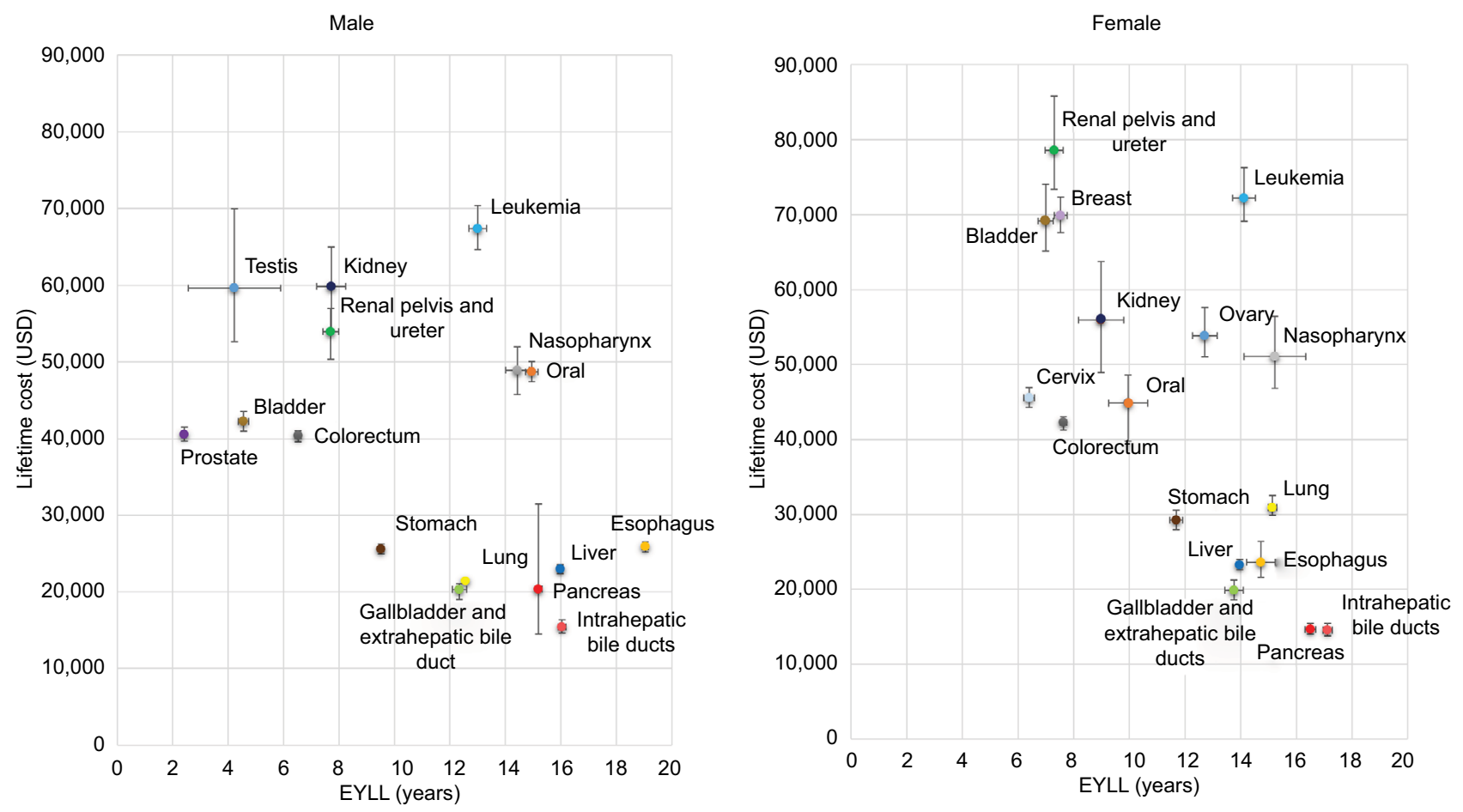

Figure $3 \mathrm{EYLL}$ and lifetime costs of the 19 major cancers in Taiwan, specified by gender. Abbreviation: EYLL, expected years of life lost. 

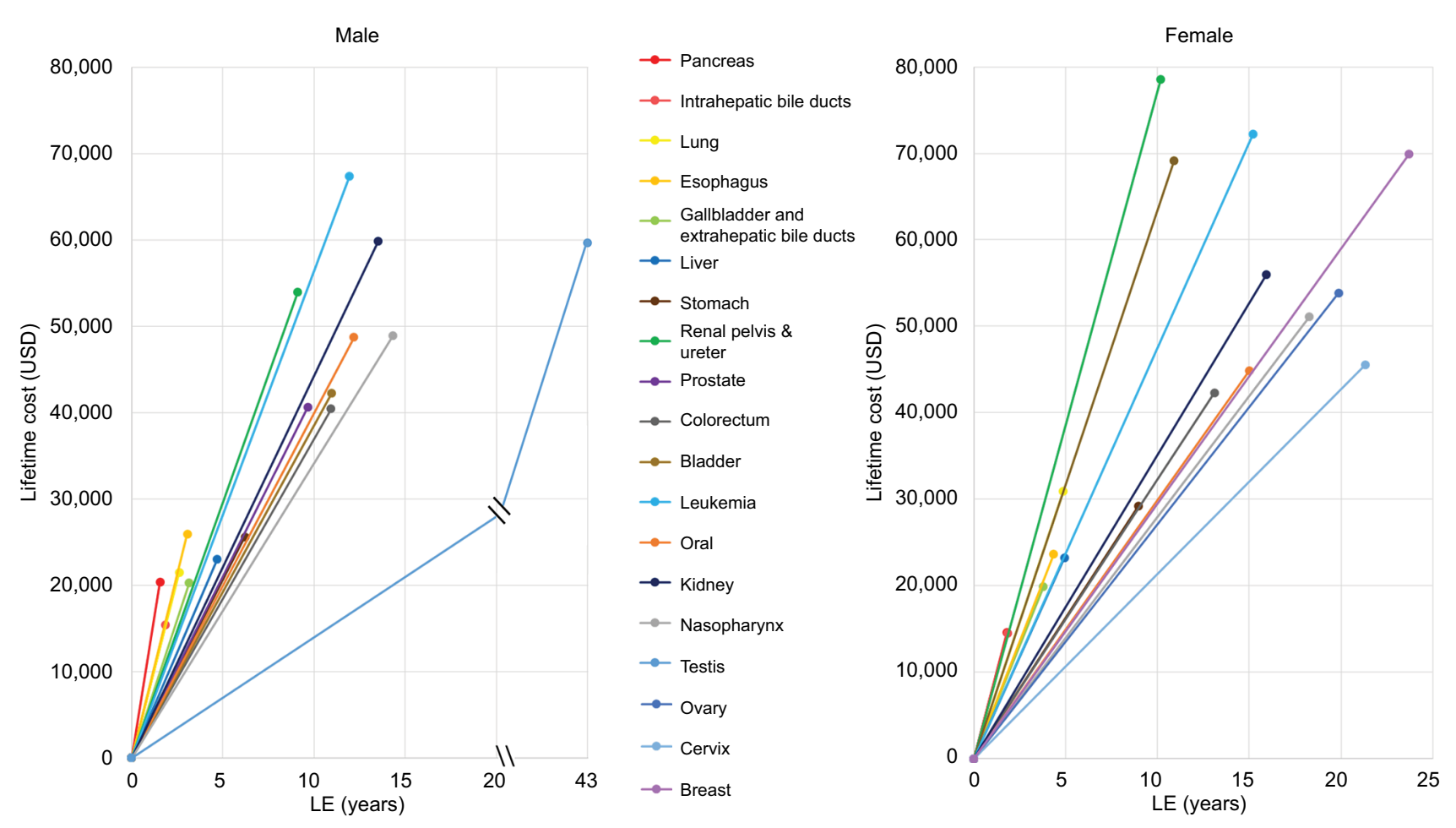

Figure 4 Generalized cost-effectiveness assessment with cost-per-life-year as the basic unit for treating major cancers in Taiwan.

Notes: By plotting the LE after diagnosis against lifetime health expenditures, this figure shows that the steeper the slope, the more expensive the treatment.

Abbreviation: LE, life expectancy.

Considering the lifetime health burden, our results showed that esophageal and intrahepatic bile duct cancers had the highest EYLL in both males and females, with estimates of 19.1 and 17.1 years, respectively. We found leukemia in males and cancer of the renal pelvis and ureter in females represented the highest lifetime costs, with estimates of US\$ 67,388 and US\$ 78,586, respectively (Table 1). In this study, however, we proposed considering both EYLL and lifetime costs together (Figure 3) as the potential savings that successful prevention of a cancer case could achieve. We found that the occurrence of one of following cancers would result in a loss of $>10$ years of EYLL plus expected health care costs of $>$ US \$ 50,000: leukemia in males and leukemia, nasopharyngeal, and ovary cancers in females.

In fact, the estimated EYLL at different stages of cancer could be used for adjustment of lead time bias on the evaluation of effectiveness of cancer screening. ${ }^{29}$ Moreover, while Figure 3 reminds us that there is a lifelong horizon for quantification of benefits of prevention, Figure 4 calls for attention to cost-effectiveness, or, cost-per-life year of management/ treatment of 19 different cancers along the same metric. Future studies are warranted to multiply the dynamic changes in quality of life (QOL), human capital loss, and disability with lifetime survival function for a more comprehensive evaluation of cost-effectiveness from a societal perspective.
Our study has the following limitations: First, the lifetime cost after cancer diagnosis in this study was only estimated based on reimbursement data from Taiwan's NHI, which does not include patients' out-of-pocket payments but includes non-cancer-related health expenditures. The lifetime health expenditures of patients with a long LE after diagnosis (e.g., $>10$ years) might be overestimated, whereas the estimation of lifetime health expenditures of patients with a short LE after diagnosis, say, $<3-5$ years, would be a lower bound. Second, this work has no data related to losses from a societal perspective, such as losses due to reduced productivity and consumption unrelated to health, as well as the other costs associated with long-term care. ${ }^{7}$ We have thus heavily underestimated the potential savings for the successful prevention of a single case of cancer. Future studies are thus warranted to attempt estimation of these societal costs. Third, we did not consider patients' QOL in the quantification of health. Since most cancers will lead to impaired QOL throughout the patient's lifetime, we have obviously underestimated the savings with regard to improved QOL due to prevention. As such, the results of this study can only be considered as a lower bound, and the cost-per-life year cannot be directly compared with cost-per-quality-adjusted life year. Although decision makers may conduct sensitivity analyses by putting different overall utility values for QOL, comprehensive assessments of QOL 
for cancers of different organ systems at different stages of cancer care are still needed for resources allocation. We may also consider quantification of loss-of quality-adjusted LE as an outcome measurement to adjust for any biased comparison related to different age distributions. Fourth, although this study followed all the cancer cohorts for 17 years, we had to rely on extrapolation to obtain the lifetime survival functions for those with a longer LE, such as patients with leukemia, cancer of the breast, cervix, and testis (Table 1). We have shown in our previous studies that our estimates would be generally more accurate than those obtained with the usual parametric method by assuming a constant excess hazard after the end of follow-up. ${ }^{30}$ Recently, we have adopted a rolling extrapolation method that would probably be the most accurate for censored claim data when estimating lifetime costs. ${ }^{11}$

\section{Conclusion}

Because big data is now becoming more accessible, we can seize the opportunity to quantify the losses of life-years and health care costs in order to improve the fairness and efficiency of cancer control efforts. Given the limited data and underestimation of social costs, we have shown that the overall impacts of cancer in terms of lifetime risks, LE, EYLL, and reimbursement costs on NHI are tremendous. In other words, cancer control strategies should consider prevention first. Future studies are warranted to stratify the health care expenditures according to cancer relatedness and item cost for different technologies as well as to take into account impaired QOL, 5-year age strata, different calendar periods, pathological stages, and various other factors based on societal perspectives, including at least functional disability and productivity loss, for a more comprehensive assessment of the cost-effectiveness of cancer control.

\section{Acknowledgments}

This work was funded by the "Multidisciplinary Health Cloud Research Program: Technology Development and Application of Big Health Data" of Academia Sinica (Taiwan) and the Ministry of Science and Technology (MOST105-2314-B-006-032, MOST106-2627-M-006-015), for which the data was obtained from the Health and Welfare Data Science Center (H104123).

\section{Disclosure}

The authors report no conflicts of interest in this work.

\section{References}

1. Torre LA, Siegel RL, Ward EM, Jemal A. Global cancer incidence and mortality rates and trends-an update. Cancer Epidemiol Biomarkers Prev. 2016;25:16-27.
2. International Agency For Research on Cancer. World Cancer Report 2014. Stewart BW, Wild C, editors. Lyon: International Agency for Research on Cancer, 2014.

3. Bray F, Jemal A, Grey N, Ferlay J, Forman D. Global cancer transitions according to the Human Development Index (2008-2030): a populationbased study. Lancet Oncol. 2012;13:790-801.

4. Bluethmann SM, Mariotto AB, Rowland JH. Anticipating the "Silver Tsunami": Prevalence Trajectories and Comorbidity Burden Among Older Cancer Survivors in the United States. Cancer Epidemiol Biomarkers Prev. 2016;25(7):1029-1036.

5. National Health Insurance Administration (Taiwan). Tables of effective number of Catastrophic Illness Card in National Health Insurance by 2017. Available from: https://dep.mohw.gov.tw/DOS/cp-3621-38058113.html. Accessed November 1, 2017.

6. National Health Insurance Administration (Taiwan). Tables of effective number of Catastrophic Illness Card in National Health Insurance by 2003. Available from: https://dep.mohw.gov.tw/DOS/cp-2916-32950113.html. Accessed November 1, 2017

7. Sanders GD, Neumann PJ, Basu A, et al. Recommendations for conduct, methodological practices, and reporting of cost-effectiveness analyses: second panel on cost-effectiveness in health and medicine. JAMA. 2016;316:1093-103.

8. Murray CJ, Lopez AD. The Global Burden of Disease. Cambridge: Harvard School of Public Health for the World Health Organization and the World Bank; 1996.

9. Liu PH, Wang JD, Keating NL. Expected years of life lost for six potentially preventable cancers in the United States. Prev Med. 2013;56:309-313.

10. Burnet NG, Jefferies SJ, Benson RJ, Hunt DP, Treasure FP. Years of life lost (YLL) from cancer is an important measure of population burden-and should be considered when allocating research funds. $\mathrm{Br}$ J Cancer. 2005;92:241-245.

11. Hwang JS, Hu TH, Lee LJH, Wang JD. Estimating lifetime medical costs from censored claims data. Health Econ. 2017;26:e332-e344.

12. Hung MC, Hsieh CL, Hwang JS, Jeng JS, Wang JD. Estimation of the long-term care needs of stroke patients by integrating functional disability and survival. PLoS One. 2013;8:e75605.

13. Hung MC, Sung JM, Chang YT, Hwang JS, Wang JD. Estimation of physical functional disabilities and long-term care needs for patients under maintenance hemodialysis. Med Care. 2014;52:63-70.

14. Day N. A new measure of age standardized incidence, the cumulative rate. In: Waterhouse JW, Muir CS, Correa P, Powell J, editors. Cancer Incidence in Five Continents. Lyon: International Agency for Research on Cancer (IARC Scientific Publications No. 15); 1976:443-445.

15. iSQoL2 Package for Windows [webpage on the Internet]. 2017 [cited 2017 9/10]. Available from: http://sites.stat.sinica.edu.tw/isqol/.

16. Taiwan Cancer Registry. Introduction of Taiwan Cancer Registry. 2017; Available from: http://tcr.cph.ntu.edu.tw/main.php?Page=A2. Accessed September 27, 2017.

17. Chiang C-J, You S-L, Chen C-J, Yang Y-W, Lo W-C, Lai M-S. Quality assessment and improvement of nationwide cancer registration system in Taiwan: a review. Jpn J Clin Oncol. 2015;45:291-296.

18. National Health Research Institute. Guidelines of quality assurance of cancer diagnosis and treatment $(Q \& A)$. Available from: http:// tcog.nhri.org.tw/accredit/Q\&A_basic2.html. Accessed November 1, 2017.

19. Rothman KJ. Modern Epidemiology. 3rd ed. Greenland S, Lash TL, editors. Philadelphia: Wolters Kluwer Health/Lippincott Williams \& Wilkins; 2008.

20. Jung K-W, Won Y-J, Kong H-J, Oh C-M, Lee DH, Lee JS. Cancer Statistics in Korea: incidence, mortality, survival, and prevalence in 2011. Cancer Res Treatm. 2014;46:109-123.

21. Siegel RL, Miller KD, Jemal A. Cancer Statistics, 2017. CA Cancer J Clin. 2017;67:7-30.

22. Lansdorp-Vogelaar I, Knudsen AB, Brenner H. Cost-effectiveness of colorectal cancer screening-an overview. Best Pract Res Clin Gastroenterol. 2010;24:439-449. 
23. Hung MC, Liu MT, Cheng YM, Wang JD. Estimation of savings of life-years and cost from early detection of cervical cancer: a followup study using nationwide databases for the period 2002-2009. BMC Cancer. 2014;14:505.

24. Chen PC, Lee JC, Wang JD. Estimation of life-year loss and lifetime costs for different stages of colon adenocarcinoma in Taiwan. PLoS One. 2015;10:e0133755.

25. Health Promotion Administration of Ministry of Health and Welfare. Taiwan Breast Cancer, Oral Cancer, and Colorectal Cancer Screening Programs. 2016. Available from: https://www.hpa.gov.tw/Pages/Detail. aspx?nodeid=1051\&pid=5957. Accessed September 27, 2017.

26. Health Promotion Administration of Ministry of Health and Welfare in Taiwan. Screening rates of women aged 30-69 receiving cervical cancer screening in recent three years (from 2000-2013). Available from: https://data.gov.tw/dataset/14673. Accessed February 28, 2018.
27. Health Promotion Administration of Ministry of Health and Welfare. Screening rates of people aged 50-69 receiving colon cancer screening in recent two years (2004-2013). Available from: http://210.71. 254.151/dataset/143070675847/resource/39ef04e8-6fdc-4902-87ec03afb6a56007. Accessed February 28, 2018.

28. Health Promotion Administration of Ministry of Health and Welfare in Taiwan. Screening rates of women aged 45-69 receiving breast cancer screening in recent two years (from 2004-2013). Available from: http://210.71.254.151/dataset/143070580718/resource/c3a2c286-bc26447f-8049-444fe696878a. Accessed February 28, 2018.

29. Yang SC, Lai WW, Lin CC, et al. Cost-effectiveness of implementing computed tomography screening for lung cancer in Taiwan. Lung Cancer. 2017;108:183-191.

30. Fang CT, Chang YY, Hsu HM, et al. Life expectancy of patients with newly-diagnosed HIV infection in the era of highly active antiretroviral therapy. $Q J$ Med. 2007;100:97-105. 


\section{Supplementary materials}

\section{Rolling extrapolation method for estimating survival}

The rolling extrapolation algorithm proposed by Hwang et $\mathrm{al}^{1}$ for estimating survival function beyond the maximum follow-up consists of 4 main steps. First, the survival times of referents are generated to match the patients in the index cohort with onset age and sex using national vital statistics. The estimated survival functions of the reference population and the index cohort are denoted as $S(t \mid$ ref $)$ for $t=0,1, . ., L$ and $S(t \mid$ ind $)$ for $t=0,1, ., F$, respectively, where $F$ is the maximum follow-up (months) and $L$ is the maximum lifespan (months) since onset age. Second, the ratio of survival functions between the index group and reference population is calculated, and denoted as $W(t)$, which is usually between 0 and 1. The logit transformation of the relative survival $W(t)$ would straighten the curve of logit $W(t)$ after a certain time point if the patients had excess constant hazard over time. The logit $W(t)$ is often smooth and approximately linear within each short time interval even when the assumption of excess constant hazard is not satisfied. Third, with the linearity property of the logit transformed curve, a restricted cubic splines model is fitted, which is linear beyond the last knot, to the observed curve of logit $W(t)$. The first 1-month ahead prediction of logit $\hat{W}(F+1)$ should be quite accurate and can be treated as a new "observed value". In the fourth step of rolling extrapolation, the data of logit $\hat{W}(t)$ is updated by dropping the first logit $W(t)$ and adding the new "observed value," and a restricted cubic splines model is re-fitted to the updated data for predicting the next logit $\hat{W}(t)$ step-by-step until $t=L$. The survival function of the index cohort beyond the maximum follow-up can be obtained by:

$$
\begin{array}{r}
\hat{S}(t \mid \text { ind })=\hat{S}(t \mid \text { ref }) \times \frac{\exp (\operatorname{logit} \hat{W}(t))}{1+\exp (\operatorname{logit} \hat{W}(t))} \\
\text { for } t=F+1, F+2, \ldots, L .
\end{array}
$$

The area between the complete survival curves of the reference population and index cohort is an estimate of the EYLL. The standard error and $95 \%$ confidence interval of the expected years of life lost estimate can be obtained using bootstrap methods. An R package iSQoL2 is available at http://www.stat.sinica.edu.tw/isqol for users to implement the calculation in practice.

\section{Brief introduction of the 4 mandated datasets (the Taiwan Cancer Registry, the Taiwan National Vital Statistics, the reimbursement database of the National Health Insurance [NHI] system, and the Taiwan Mortality Registry) used in this study}

1. Taiwan Cancer Registry: The cancer diagnosis must be first validated by 2 qualified physicians, which are recorded into the registry by 2 trained health care professionals who have been certified to perform such a task. The data cannot be formally stored in the dataset until all logical errors are corrected and/or debugged. ${ }^{2,3}$

2. Taiwan National Vital Statistics: It was first established in 1905. After several decades of improving the quality, it has provided highly accurate and reliable demographic information and vital statistics for population resided in Taiwan. The algorithm of national vital statistics was updated in 1998 to more precisely match every detail of the population structure. ${ }^{4}$

3. The reimbursement database of the NHI system: The reimbursement database of the NHI system covers $>99.9 \%$ of people in Taiwan. Moreover, the committee of NHI reimbursement reviews the data and debugs unreasonable expenditure every 3 months (https://nhird.nhri.org.tw/en/). ${ }^{5}$

4. Taiwan Mortality Registry: This is based on records from death certificates, which must be issued by doctors, although some are provided by prosecutors, especially those who committed suicide or died of injuries. The family and related institutions will be imposed with a fine if they do not report a death. ${ }^{6}$

In summary, the quality of these 4 datasets are highly reliable and valid.

\section{References}

1. Hwang JS, Hu TH, Lee LJ, Wang JD. Estimating lifetime medical costs from censored claims data. Health Econ. 2017;26:e332-e344.

2. Chiang CJ, You SL, Chen CJ, Yang YW, Lo WC, Lai MS. Quality assessment and improvement of nationwide cancer registration system in Taiwan: a review. Jpn J Clin Oncol. 2015;45:291-296.

3. Taiwan Cancer Registry. Introduction of Taiwan Cancer Registry. Available from: http://tcr.cph.ntu.edu.tw/main.php?Page=A2. Accessed September 27, 2017.

4. Ministry of Interior, Republic of China. Demographic Facts, 1968-1982. Taipei: Ministry of Interior, 1969-1983.

5. National Health Insurance Administration, Ministry of Health and Welfare, Taiwan, R.O.C. National Health Insurance Annual Report 2014-2015; 2014.

6. Ministry of Welfare and Health. Handbook of cause of death dataset. Available from: $\mathrm{http} / /$ dep.mohw.gov.tw/DOS/lp-3147-113.html. Accessed February 28, 2018. 


\section{Colorectal cancer}

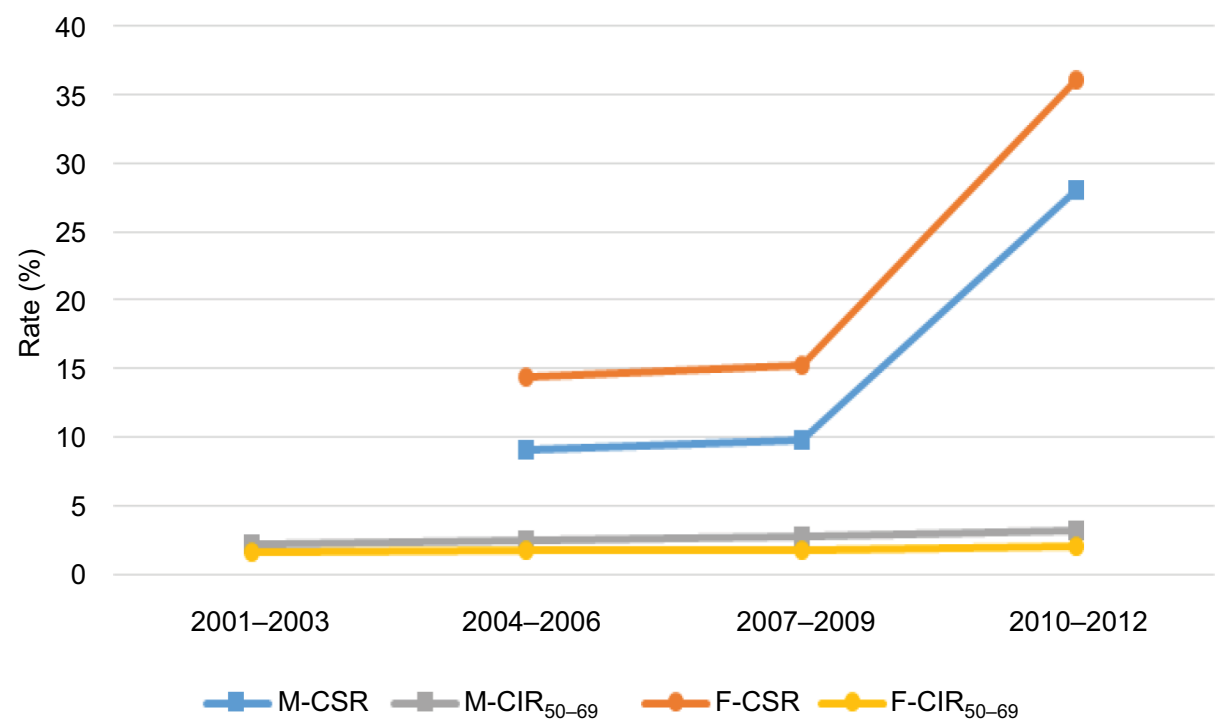

Figure SI Comparison of the trends of cancer screening rate and cumulative incidence rate of colorectal cancer every 3 years.

Abbreviations: M-CSR, cancer screening rate of male; $M-C_{12}{ }_{50-69}$, cumulative incidence rate for males aged 50-69 years; F-CSR, cancer screening rate of female; F-CIR $50-69$, cumulative incidence rate for females aged 50-69 years.

Female breast cancer

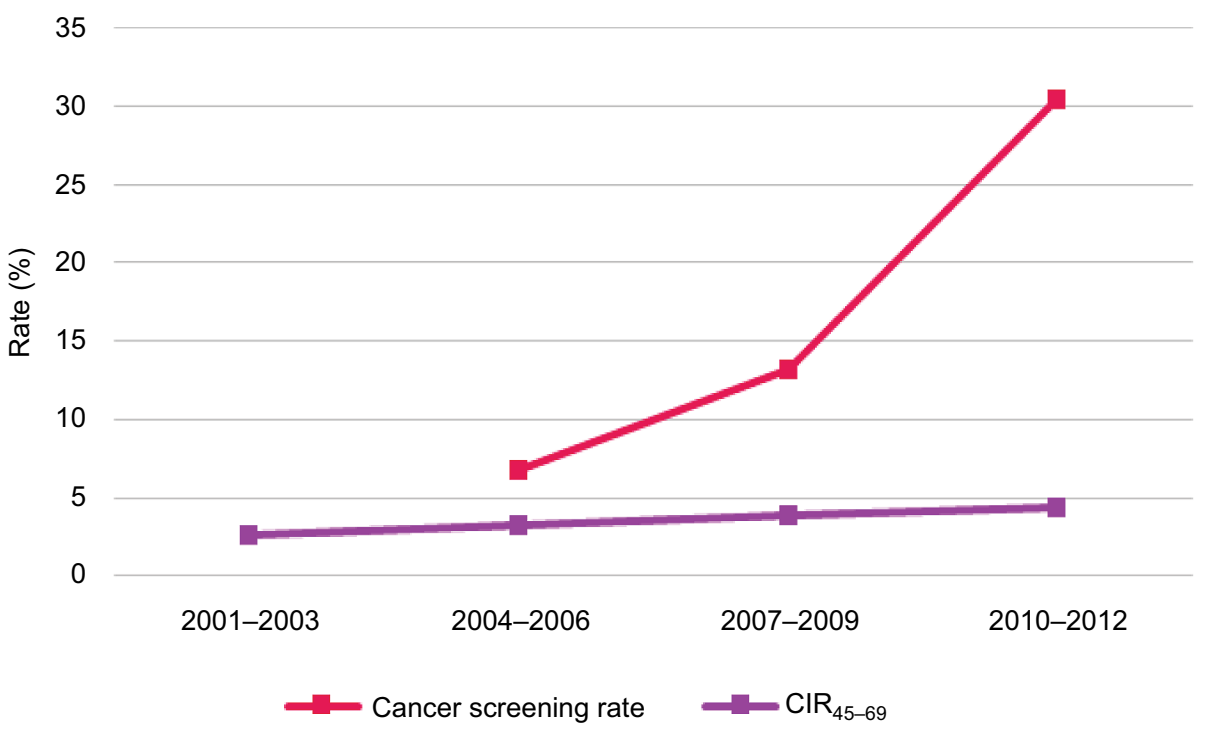

Figure S2 Comparison of the trends of cancer screening rate and cumulative incidence rate of breast cancer along calendar periods. Abbreviation: $\mathrm{CIR}_{45-69}$, cumulative incidence rate for women aged $45-69$ years.

Clinical Epidemiology

\section{Publish your work in this journal}

Clinical Epidemiology is an international, peer-reviewed, open access, online journal focusing on disease and drug epidemiology, identification of risk factors and screening procedures to develop optimal preventative initiatives and programs. Specific topics include: diagnosis, prognosis, treatment, screening, prevention, risk factor modification,

Submit your manuscript here: https://www.dovepress.com/clinical-epidemiology-journal

\section{Dovepress}

systematic reviews, risk and safety of medical interventions, epidemiology and biostatistical methods, and evaluation of guidelines, translational medicine, health policies and economic evaluations. The manuscript management system is completely online and includes a very quick and fair peer-review system, which is all easy to use. 